\title{
Unstructured Grid Generation for Complex 3D High-Lift Configurations
}

\author{
Shahyar Z. Pirzadeh \\ NASA Langley Research Center, Hampton, VA 23681-2199
}

\begin{abstract}
The application of an unstructured grid methodology on a three-dimensional high-lift configuration is presented. The focus of this paper is on the grid generation aspect of an integrated effort for the development of an unstructured-grid computational fluid dynamics (CFD) capability at the NASA Langley Research Center. The meshing approach is based on tetrahedral grids generated by the advancing-front and the advancing-layers procedures. The capability of the method for solving high-lift problems is demonstrated on an aircraft model referred to as the energy efficient transport configuration. The grid generation issues, including the pros and cons of the present approach, are discussed in relation to the high-lift problems. Limited viscous flow results are presented to demonstrate the viability of the generated grids. A corresponding NavierStokes solution capability, along with further computations on the present grid, is presented in a companion SAE paper.
\end{abstract}

\section{INTRODUCTION}

One of the most challenging applications of CFD concerns the computation of fluid flow around aircraft in the take-off and landing configurations. Besides the complexity of the flow physics involved (usually characterized by strong viscous-dominated flow features), the geometric complexity of the high-lift configurations makes the routine application of the advanced CFD methods difficult for this class of problems.

High-lift problems typically feature geometric complexities such as multiple components, small gaps between various elements, sharp edges and corners, etc. These characteristics, along with other geometry processing complications such as inadequately defined computer-aided-design (CAD) surfaces, make the task of generating suitable CFD grids extremely difficult and time-consuming. The extensive amounts of grid generation time and engineering skill required are major factors which impede the practical use of the advanced CFD tools for high-lift problems. The challenge is further amplified in a multi-disciplinary design environment in which multiple layers of analyses are required for a complete design cycle within a short period of time.

As mentioned earlier, "viscous" effects usually dominate flowtields in the high-lift problems. Therefore, the Navier-Stokes ( $N-S$ ) equations should be solved to simulate the complex flowfields accurately. Resolving the boundary layer with grid elements of high-aspectratio presents stringent requirements and poses increased difficulties for generating appropriate grids. Furthermore, high-lift problems usually feature many complex flow phenomena, each requiring elaborate grid specifications. Among the dominant flow features are over expansion of flow in the small gaps between the high-lift wing elements that may lead to small shock waves, shock/boundary-layer interactions, massive flow separations, extended turbulent eddies in wake of the wing elements (affecting flow on other elements downstream), and vortices emerging from the tips of the wing elements. Since many of these flow phenomena interact with each other, failing to predict one could adversely affect the prediction of others in the adjacent regions. This, in turn, would propagate solution errors throughout the flowtield. To achieve the desired level of accuracy, proper distribution of grid nodes at the appropriate locations is essential for resolving these dominant flow features. As the location and extent of these flow phenomena are usually unknown in advance, the need for an automatic adaptive grid methodology is apparent.

A majority of CFD research on the high-lift problems has been performed on two-dimensional (2D) cases as reported in the literature (see, for example, Refs. 1-5). A 2D approach provides useful information on the basic flow characteristics conveniently and expeditiously. However, it is obvious that a realistic picture of the complex high-lift flowtield can only be portrayed in the three-dimensional (3D) space. Evidently, fewer attempts have been made to address the problem in 3D mainly due to complexity of the grid generation involved. Among the $3 \mathrm{D}$ results reported, many concern the computation of relatively simple high-lift configurations such as isolated wings with fully or partially deflected flaps. ${ }^{6-8}$ One of the initial attempts to compute the highlift problem on a transport configuration is that made by Dodbele ${ }^{9}$ using an unstructured grid methodology. The flow computation, presented in that work, is inviscid and, thus, lacks the desired accuracy. 
An outstanding effort by Rogers et $\mathrm{al}^{10}$ and Cao et $\mathrm{al}^{\text {"1 }}$ concerns the computation of a complete Boeing 747PD high-lift configuration using an overset-grid approach. The reports elaborate on an overset grid methodology, as applied to the 747PD configuration, as well as the corresponding N-S solutions. The work has successfully demonstrated that the computation of complex realistic high-lift problems is now achievable with the current CFD capabilities. However, the reports also draw two important conclusions regarding the implementation of the methodology for the high-litt problems. First, it has taken several months to prepare a grid system for the high-lift configuration with the technology utilized. Such a time frame is obviously unacceptable for a design process. Secondly, errors due to the interpolation of flow solutions between cells of large volume disparities in an overset grid contribute to the solution inaccuracy. For a complex multi-element high-lift configuration, it is difficult to remove such resolution disparities among the overlapped grids.

An unstructured-grid, N-S computation of a high-lift configuration referred to as the Energy Efficient Transport (EET) has recently been reported by Mavriplis and this author. ${ }^{12}$ An attractive feature of the methodology employed is the speed by which a complete "geometry to drag-polar" analysis of a complex 3D high-lift problem can be performed. It has been demonstrated, in that work, that unstructured grids of several million cells can be constructed for complex, high-lift geometries on workstations in a matter of days. In addition, flow solutions can be generated on the existing production supercomputers in several hours.

The unstructured grid technology offers a substantial advantage over the conventional structured grid methods for handling complex geometries. Due to their inherent flexibility, unstructured grids conform to complex geometries conveniently and, thus, are generated in a considerably reduced amount of time and effort. In addition to the ease of generation, unstructured grids offer convenience for the effective distribution of grid points, solution adaptive refinement, local re-meshing, and the incorporation of moving boundaries. A disadvantage of the unstructured grid methods (especially those based on tetrahedral or mixed element meshes) is their large requirements for computational resources (speed and memory). Therefore, the implementation of parallel computing appears to be inevitable for unstructured-grid N-S solutions of large problems. Another bottleneck for generating grids, in general, is the construction of "water-tight" surface definitions from the CAD geometry models. High-lift configurations contain many components, each comprising of several (often mismatched) surface definitions. The process of surface preparation is, for the most part, manual and timeconsuming which, at the best, takes many hours or days of surface patching and assembly and, at the worst, requires several weeks of geometry repair and reconstruction.

The objective of this paper is to demonstrate the capability of an unstructured grid generation system, known as VGRIDns, for the generation of thin-layered tetrahedral "viscous" grids around complex high-lift configurations. The focus of the paper is primarily on the grid generation aspect of the work reported in Ref. [12]. A brief discussion of the grid generation procedure, as applied to the EET configuration, is presented in this paper. The difference between the present problem and the one in Ref. [12] is the addition of a pylon and nacelle to the EET geometry for this study. The computational results of an unstructured grid N-S solution technique on the present grid are presented in a companion paper by - Mavriplis. ${ }^{13}$ A sample flow solution, taken from that report, is also included in the present paper for completeness.

\section{GRID GENERATION APPROACH}

The computational grid presented in this paper has been generated using the unstructured grid generation system VGRIDns ${ }^{14}$. The system, developed at the NASA Langley Research Center, includes the grid utility software GridTool $^{15}$, the unstructured grid generator VGRIDns, and the grid post-processing utility code POSTGRID. Among the recent developments are the solution adaptive and moving grid capabilities ${ }^{16}$. The implementation of these capabilities into the VGRIDns system is currently under way. The process of grid generation with VGRIDns is described in the following sections, from surface definition to the final postprocessed volume grid as applied to the EET configuration.

\section{GEOMETRY DESCRIPTION}

The Energy Efficient Transport is an unpowered, twin engine, wind tunnel model in a take-off configuration. For computational economy, a semi-span model of the geometry is considered as the test case for the present study. The subject geometry consists of a semi-span fuselage of 9.91 feet long, a super-critical wing with an aspect ratio of 10 and leading-edge sweep angle of 28.8 degrees, inboard and outboard leadingedge slats at -50 degrees deflection, double-slotted flaps at 30 degrees deflection with corresponding vanes at 15 degrees deflection, a pylon attached to the main wing element, and a flow-through nacelle. The model has been tested in the NASA Langley $14 \times 22 \mathrm{ft}$ subsonic wind tunnel' ${ }^{17}$. The surface $C A D$ definition, given in the standard Initial Graphics Exchange Specifications (IGES) format, has been constructed using the measured data in the Geometry Laboratory (GEOLAB) at the NASA Langley Research Center. The IGES file contains 126 surfaces and 1027 curves, as shown in Figure 1.

The geometry involves complexities such as sharp corners/edges and extremely narrow gaps. The smallest

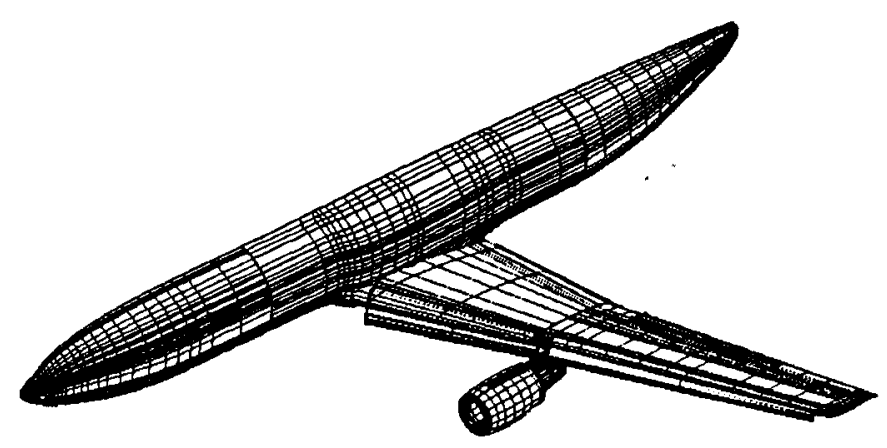

Figure 1. Surface definition (IGES) for the EET configuration. 
gap is between the side of the pylon and tip of the deflected slat with a width of about 0.027 in. Such a gap size is comparable to the boundary layer thickness at the location (the measured boundary layer thickness on the model fuselage ahead of the wing is about 0.5 in.) These geometric difficulties make the generation of good quality viscous grids extremely complicated.

\section{SURFACE GRID GENERATION}

The surface grid generation is performed in two main steps: 1) geometry set-up and preparation of grid parameters with GridTool and 2) surface triangulation with VGRIDns.

\section{Surface Patching}

A geometry model to be meshed with VGRIDns is represented by a set of independent surface patches. The union of these contiguous patches, including those defining the outer boundaries, forms a "water-tight" surface boundary enclosing the entire computational domain. A CAD model, usually defined in a standard surface definition such as the lGES format, is read into the grid utility software GridTool to construct the surface patches. This user-friendly program converts the given surface definitions into a set of internal non-uniform rational B-spline (NURBS) surfaces for convenient construction of surface patches. Using the NURBS information and many useful graphical features in GridTool, one can easily create and modify arbitrary curves and surface patches, assuming the given surface model is defined adequately. In the present example, the individually defined IGES surfaces and curves were used to construct 1462 parametric surface patches with GridTool. Many of these patches were automatically constructed with the "auto-patch" option of GridTool for generating the grid presented in Ref. [12]. For the present grid, new surface patches defining the nacelle and pylon were constructed and added to those prepared previously. The collection of all patches constitutes a solid (water-tight) surface to be used by the grid generator (see Fig. 2).

The process of preparing surface patches and grid distribution parameters (described in the next section) constitutes $50-90 \%$ of the total grid generation time depending on the complexity of the geometry and the "cleanliness" of the CAD surface definition. When translated into the data exchange standards, the CAD

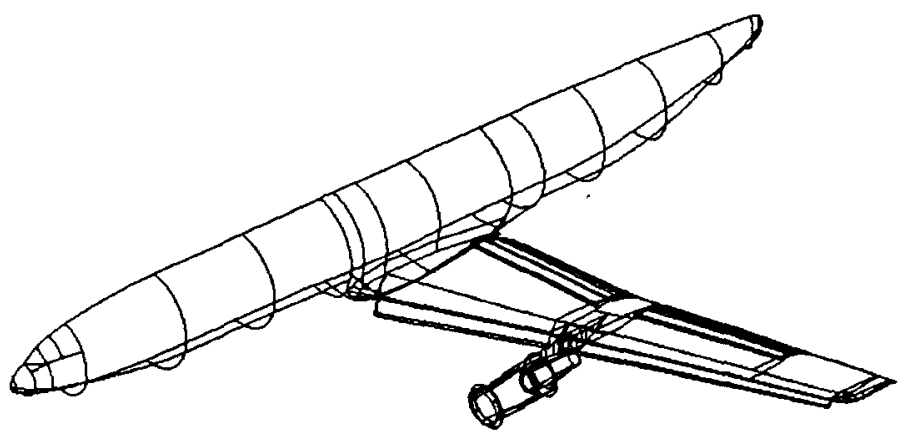

Figure 2. EET configuration defined in terms of surface patches. models usually lose their precision/integrity and produce surface representations which, in some cases, are severely distorted and difficult to work with. ${ }^{18}$ A substantial amount of grid generation time and effort is often spent to fix surface anomalies before the actual grid generation process starts.

\section{Cartesian Background Grid and Source Elements}

In the grid generation methodology utilized in VGRIDns, a smooth variation of grid characteristics in the field is crucial for the success of the process and the quality of the generated grids. In the present method, the grid characteristics such as spacing, rate of growth, and stretching parameters are prescribed through several "point" and "line" sources placed in the domain by the user. ${ }^{19}$ Associated with the source elements is a secondary "background" grid for the distribution of grid parameters in the field. In VGRIDns, a "transparent" (automatically constructed) Cartesian grid overlaying the entire domain serves as the background grid. A smooth propagation of the grid parameters in the field is achieved by solving an elliptic partial differential equation on the background grid, in analogy to a heat conduction problem, with the sources acting as heat elements. During the grid generation process, the smoothed grid parameters are interpolated from the nodes of the background grid and sources to create the unstructured grid elements.

The application of source concept for generating unstructured grids has provided two important advantages over the method originally employed. First, clustering of grid points on the surface and in the field is convenient and controllable. Secondly, the generated unstructured grids are of high quality due to the elliptic smoothing effect of the background grid. Two disadvantages of the method are the lack of automation and the inability to prescribe exact number of surface mesh points at the precise locations. The placement and adjustment of the source elements, using the present capabilities of the system, are user-interactive and require experience. The problem is especially intensive for complex problems, such as high-lift, which require many sources to produce elaborate and high-quality point distributions. A more automated technique with limited dependency on sources and based on automatic solution/geometry grid adaptation is desirable. The development of such a capability is currently in progress.

The source elements and their characteristics are

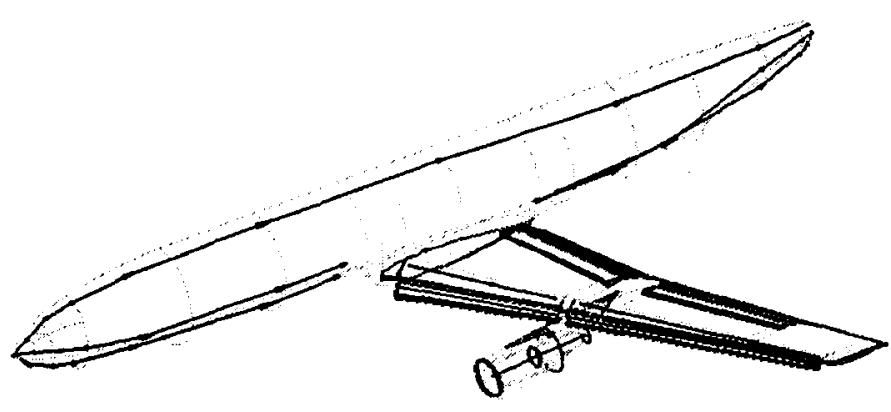

Figure 3. Background grid source elements. 
prescribed with GridTool to complete the VGRIDns input data file. To produce a high-quality point distribution on the surface and in the field of the present grid, a total of 924 sources (mostly line sources) were prescribed. Many of these sources were constructed with an automatic ("curve-to-source") option in GridTool (see Fig. 3). As mentioned earlier, the process of surface patch and source preparation takes a large portion of the total grid generation time and requires some user experience depending on complexity of the problem.

\section{Surface Triangulation}

After the input data file containing the surface patches and sources is prepared, the surface grid is generated with VGRIDns automatically. The grid generation technique used in VGRIDns is based on the advancing-front method $(A F M)^{20}$, for generating "inviscid" grids, and the advancing-layers method $(A L M)^{14}$, for producing thin layered "viscous" meshes. These methods are based on marching techniques in which new grid elements initially grow on the boundaries and gradually march in the field until the entire domain is filled with contiguous grid cells. For the surface mesh generation, each surface patch is considered as an independent domain. The edges of the patch are first discretized to form an initial front. The patch is then mapped into a computational $(u, v)$ space and meshed with the $2 \mathrm{D}$ versions of the AFM and ALM. The triangulated patch is then transformed back from the computational to the physical $(x, y, z)$ space. An assembly of all triangulated patches forms the surface mesh, which also represents the initial front for the volume grid generation.

A disadvantage of VGRIDns for generating surface grids is due to the parametric surface patches that are defined in terms of their edges only. Consequently, the position of an interior grid node on a surface patch is approximate and does not necessarily conform to the original CAD surface definition. To correct the problem, the surface mesh points must be projected onto the NURBS surfaces with GridTool. The projection process with GridTool is user-interactive and time-consuming. In

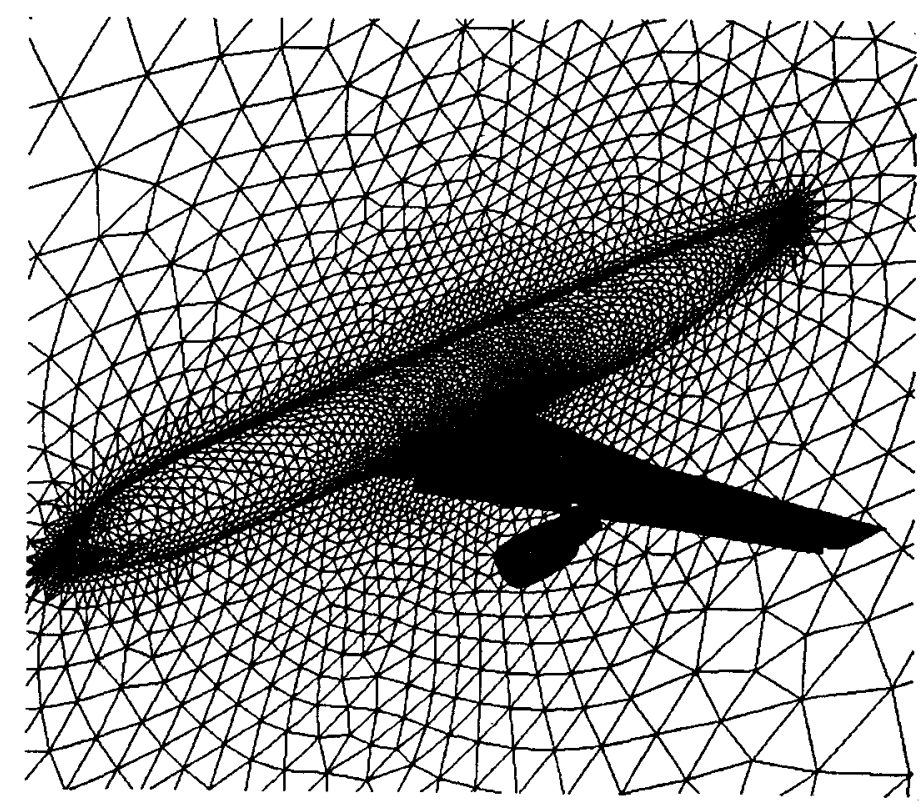

Figure 4. Surface triangulation on the EET. addition, surface grid nodes are occasionally misplaced during the projection, which if unnoticed, would create unexpected grid generation problems. Recently, a new surface mesh projector has been developed as part of an ongoing adaptive grid work, which alleviates many of the previous projection problems. The new code is based on a robust NURBS library, written by Samareh, similar to that reported in Ref. [15]. The new code projects surface grids in the batch mode automatically. Work is also underway to add to VGRIDns the capability of generating surface grids directly on the NURBS surfaces. The enhancement would eliminate the need for surface mesh projection.

Figure 4 illustrates the EET surface grid, along with the triangulation on the symmetry plane. The surface grid nodes were automatically projected onto the NURBS surfaces using the newly developed projection program. Several close-up views of the surface mesh on the fuselage, wing, flaps, nacelle, and pylon are shown in Fig. 5. These images reveal the details and complexity of the geometry. The narrow gaps among the wing, slat, and pylon are clearly visible in Fig. 5d. As evident from these pictures, the surface geometry is adequately resolved with good quality triangular mesh distribution, especially at the edges, corners, and gaps. The grid is anisotropicly stretched in the spanwise direction as indicated at the leading edge of the slat in Fig. $5 \mathrm{~d}$. Anisotropic stretching is applied for grid efficiency and is described in a separate section of this paper. The thin viscous layers and their smooth transition to the outer regular grid are visible around the geometry on the symmetry plane in Fig. $5 b$.

\section{VOLUME GRID GENERATION}

The generation of tetrahedral volume grid is achieved with the 3D versions of the marching techniques AFM and ALM, similar to those utilized for surface triangulation. The following subsections provide brief discussions on the main features of the techniques.

\section{Advancing Front Method}

During the AFM process, for generating an inviscid (or inviscid portion of a viscous) grid, tetrahedral cells start growing on the triangular faces of the surface mesh and gradually advance in the field in an irregular manner. When new cells are formed, the old faces covered with tetrahedrons are removed from the front, and new triangular faces (sides of the new tetrahedrons) are added to the list. The process continues, as the front evolves and advances in the field, until the entire domain is filled with contiguous tetrahedral cells. At this point, no "active" front face remains in the field.

The method has matured into a viable grid generation method as different investigators have added new capabilities to it over the years. Two useful features implemented in the method are the restart capability and local remeshing. A volume grid can be generated with VGRIDns in one or several restart runs. The code exploits a recurrent global/local renumbering procedure ${ }^{21}$ that reduces the computer memory requirement for generating large size grids (such as those presented in this paper) on workstations with limited core memory. A local remeshing capability has also been implemented for grid post-processing ${ }^{21}$ and adaptive grid refinement. ${ }^{16}$ 


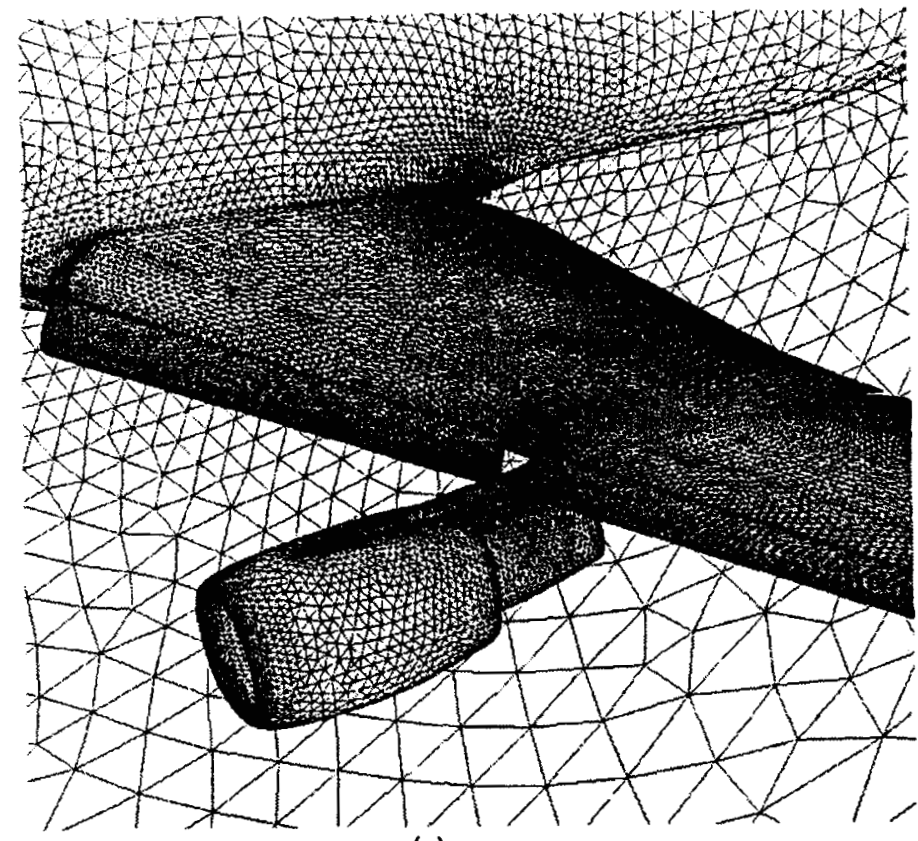

(a)

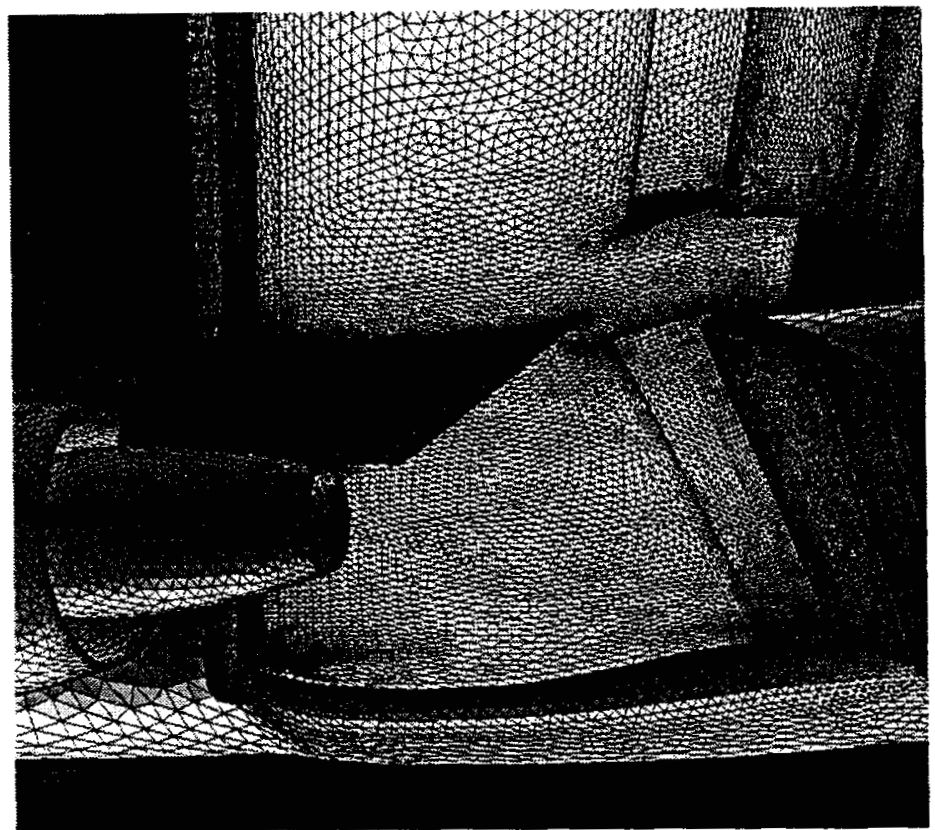

(C)

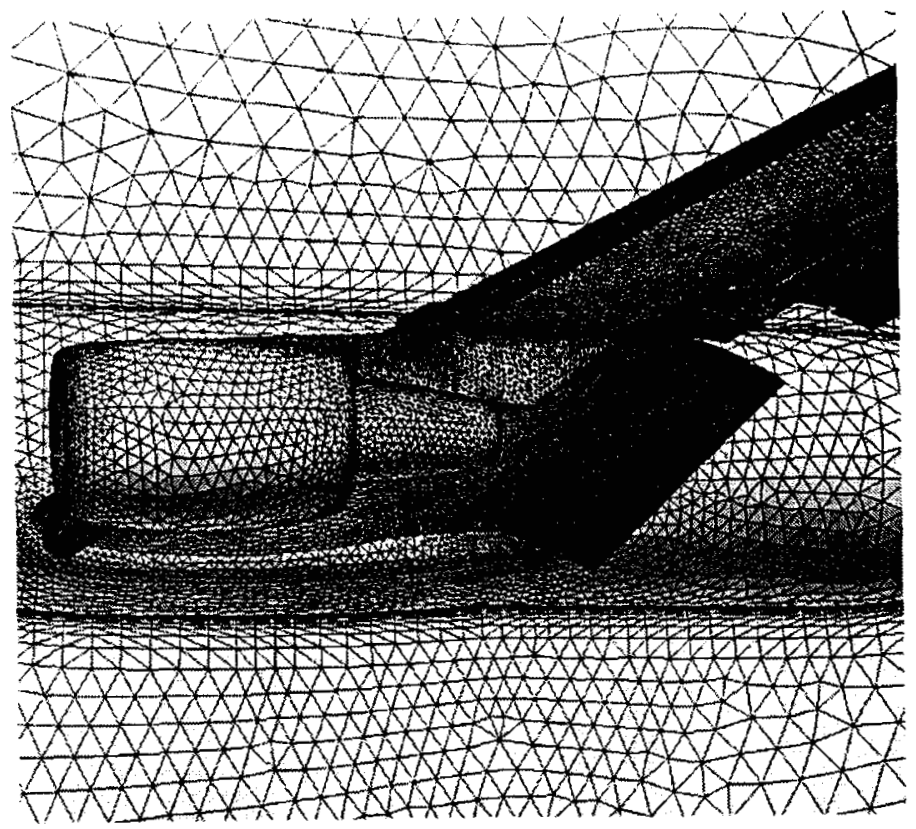

(b)

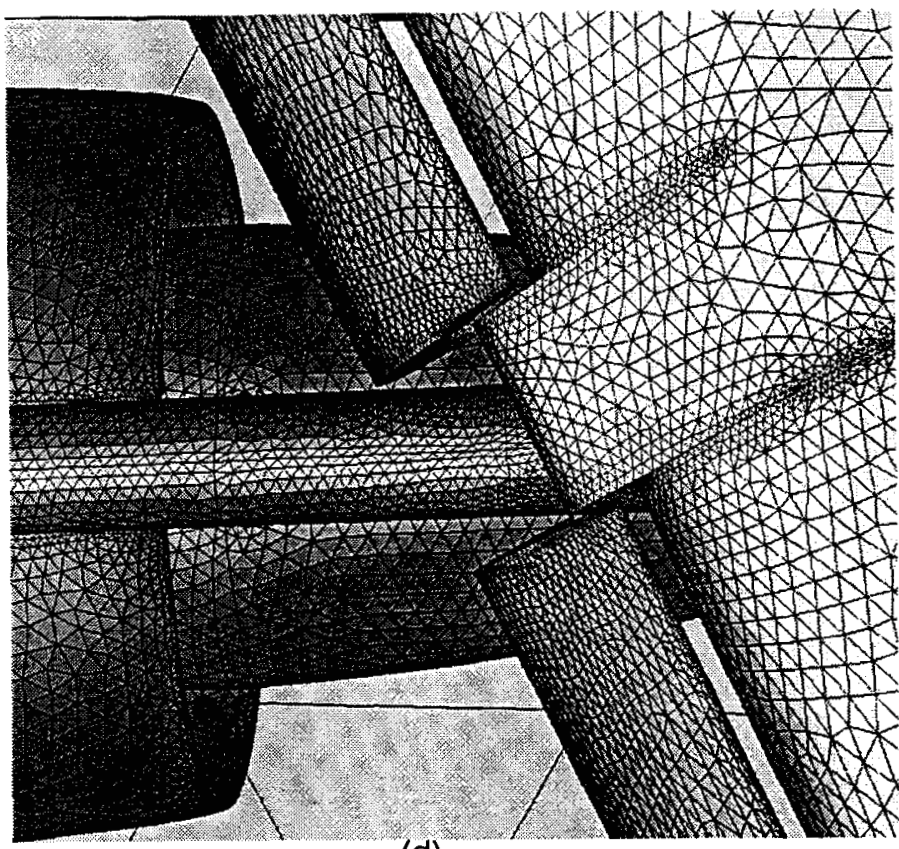

(d)

Figure 5. Close-up views of the surface mesh: (a) wing-pylon-nacelle (upper surface), (b) wing-pylon-nacelle (lower surface), (c) and (d) details of the pylon-slat region showing small gaps between components.

\section{Advancing Layers Method}

Although the AFM is a proven technology for generating regular (inviscid) tetrahedral grids, it is not suitable for constructing high-aspect-ratio cells to resolve the boundary layer. Instead, a similar approach, referred to as the advancing-layers method, has been developed to generate grids in the viscous dominated regions close to the solid surfaces. Once the boundary layer is meshed, the conventional AFM is applied to complete the grid in the rest of the domain. Unlike the conventional AFM, which introduces cells in the field in an unstructured fashion (no apparent order), the ALM generates layers of thin tetrahedral cells in a more systematic manner while maintaining the flexibility of the AFM. The strategy alleviates many grid generation complexities that usually arise from the floating-point operations of small numbers associated with viscous grids.

Two main operations are involved in the ALM: 1) computation of a set of surface normal vectors along which the grid points are distributed and 2) construction of a pattern of compatible tetrahedral connectivity for the thin cells to be generated in the boundary layer. The surface vectors are calculated using an iterative algorithm based on an "equal-angle" criterion followed by a Laplacian smoothing operation. The connectivity pattern is predetermined by an efficient, all-integer algorithm that eliminates the need for a series of computer-intensive, floating-point calculations as used in the conventional AFM. Additional information about the surface vector and connectivity algorithms can be found in Ref. [14]. 


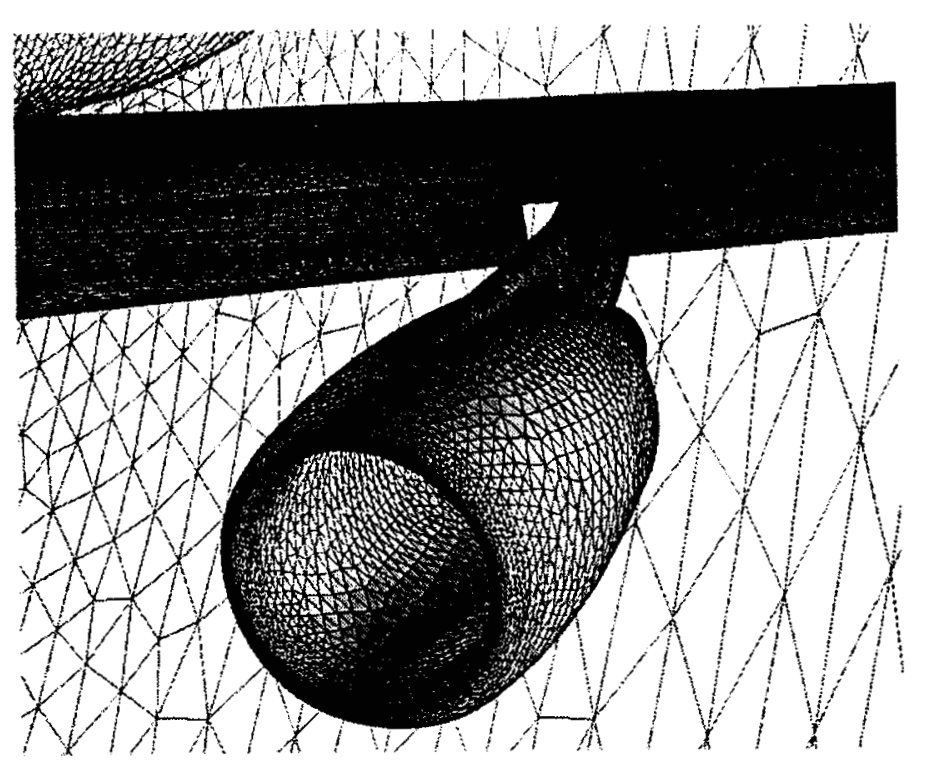

(a)

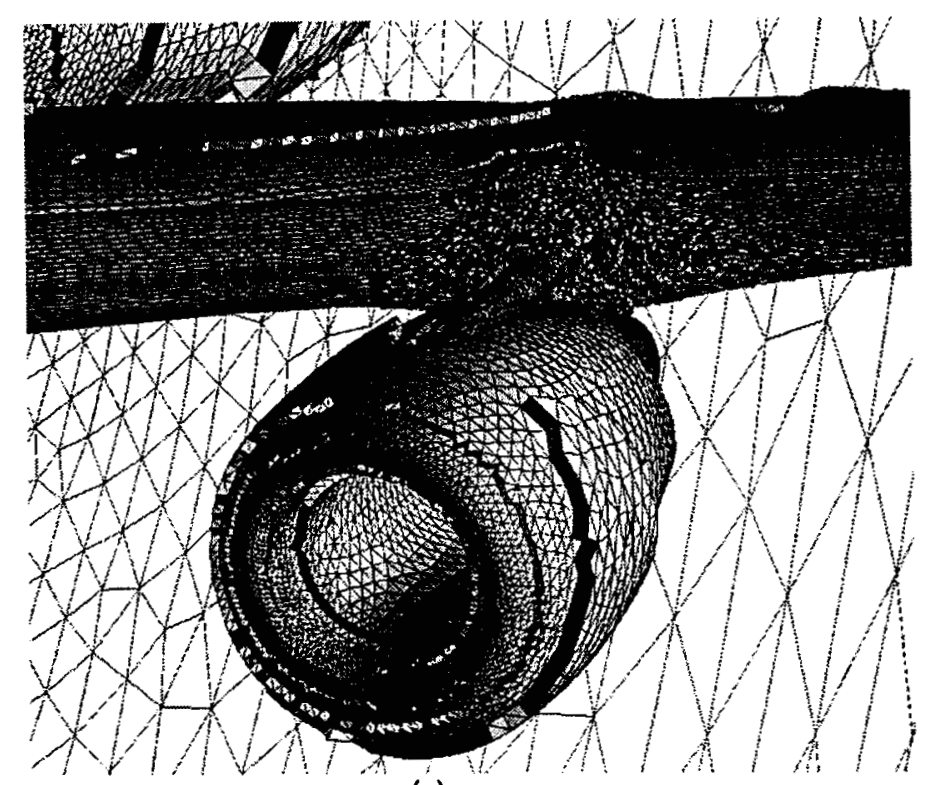

(c)

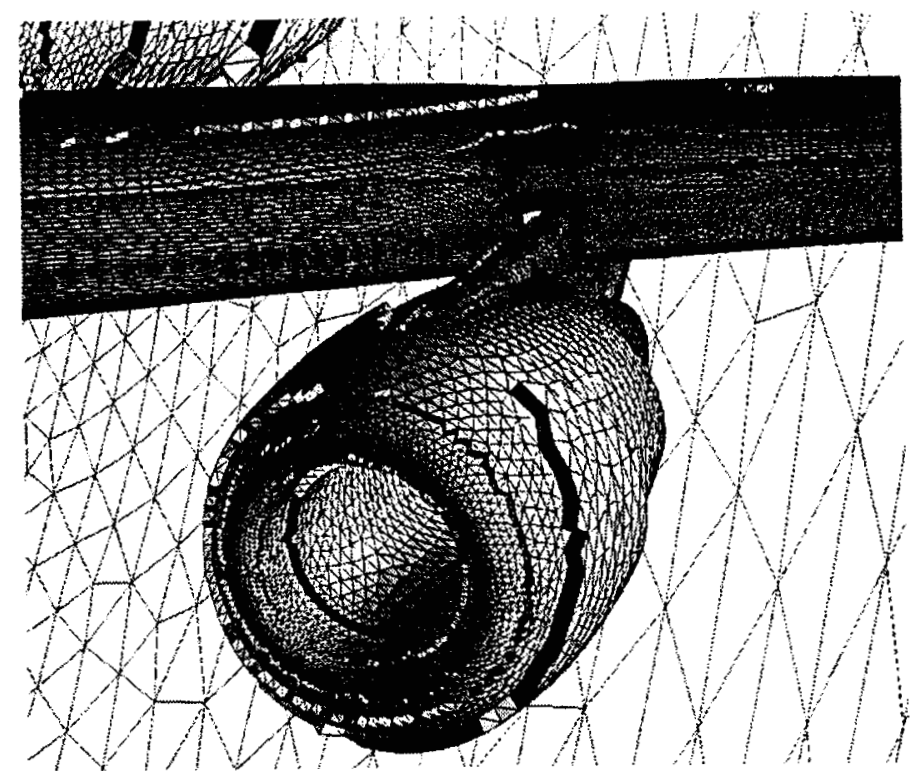

(b)

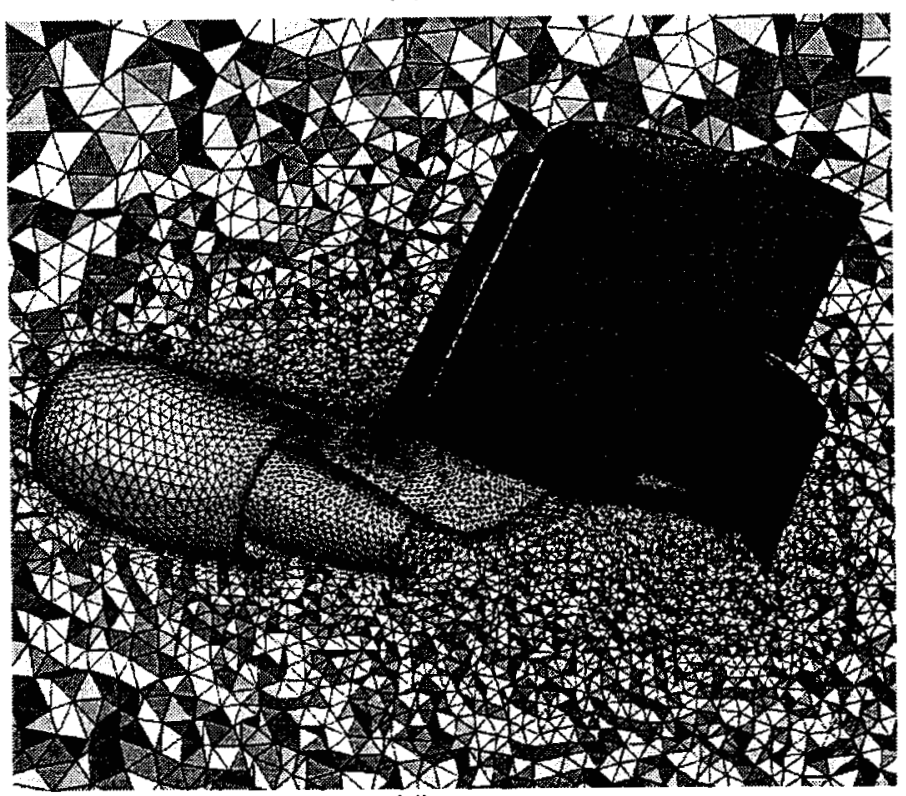

(d)

Figure 6. From surface to volume grid: (a) surface mesh, (b) viscous grid layers generated with ALM, (c) regular tetrahedrons being generated on top of viscous layers, and (d) cross-section of complete grid.

Thin layers of tetrahedral cells are constructed by inserting new points along the surface vectors and connecting them according to the predetermined connectivity pattern. The normal grid spacing along the surface vectors is determined by the stretching function

$$
\delta_{n+1}=\delta_{1}\left[1+a(1+b)^{n}\right]^{n}
$$

where $\delta_{n}$ is the normal spacing of the $n^{\text {th }}$ layer, $\delta_{1}$ is the first layer spacing prescribed by the user, and the factors $a$ and $b$ are constants that determine the rate of stretching, also prescribed by the user.

Thin tetrahedral cells grow on the surface mesh (see Fig. 6) and advance in the field one layer at a time. The layers continue marching as their heights, determined by Eq (1), increase until some limiting criteria prevent them from further advancement. Two criteria are employed for this purpose: 1) the normal spacing determined by $\mathrm{Eq}(1)$ as compared to the local background-grid spacing and 2) proximity of the front faces approaching from opposite directions. During the advancing process, layers may stop at a location, where the limiting conditions are satisfied, but continue growing at other locations resulting in "incomplete" layers (Fig. 6b). Once the layers cease to advance everywhere, the process automatically converts from the advancing-layers to the advancing-front mode to generate regular grid cells outside the boundary layer (see Fig. 6c). With a common background grid controlling both methods, the transition from the layers to the regular grid becomes smooth and continuous. In addition, the fact that the number of layers varies from one location to another adds to the flexibility of the method. This feature is particularly important for generating grids on geometries having small gaps between components and sharp corners like those present in most high-lift configurations. For more 
information on the ALM procedure, see Ref. [14]. Finally, Figure $6 d$ illustrates a cross-section of the complete volume grid cut by a plane passing through the wing components, pylon and the nacelle.

\section{Anisotropic Grid Stretching}

An important issue concerning the use of unstructured tetrahedral grids for the $\mathrm{N}-\mathrm{S}$ computations of complex high-lift configurations is the large size of the generated grids. A typical high-lift problem requires at least 10 million grid points for a wing/body/flaps geometry (according to Cao et $\mathrm{al}^{11}$ ) and 10-100 million points for a complete transport high-lift configuration (based on an estimate by Mavriplis ${ }^{12}$ ). Unstructured grid solvers usually require more computer memory per grid node than their structured grid counterparts. Consequently, advanced techniques are required to make the unstructured-grid methodology practicable for realistic high-lift problems.

As mentioned earlier, one solution is the utilization of parallel computations with grid decomposition, which has created excellent opportunity for solving "large" problems. Grid adaptation is another powerful technique to alleviate the problem by producing efficient grids and improving the solution accuracy economically. An effective approach for generating more efficient unstructured grids is to distribute points anisotropicly. Many unstructured mesh generators produce grids containing equilateral elements. These isotropic grids usually contain excessive number of points and cells in directions in which the flow variation is small.

A salient feature of VGRIDns is its ability to generate multi-directional, anisotropicly stretched grids by which surface triangles and tetrahedrons in the field are elongated in user-prescribed directions ${ }^{22}$. With this capability, fewer points are distributed in the directions of reduced flow gradient without loss of grid resolution in other essential directions. Grid stretching with VGRIDns

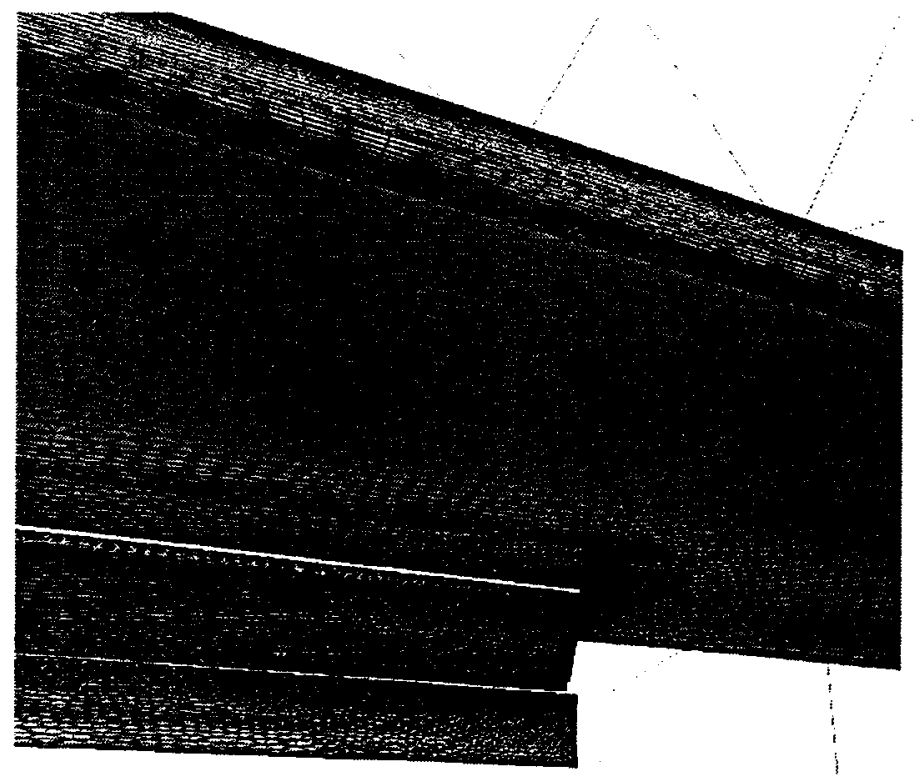

Figure 7. Anisotopic grid stretching applied at the leading edges of EET wing elements.

is achieved by prescribing a stretching vector and two spacing parameters (along and normal to the stretching vector) for each background grid source. To minimize complications that arise from the advancement of highly stretched cells in the field, a local transformation is performed for each new cell being generated. The physical (stretched) space is mapped into an isotropic frame in which equilateral grid elements are created, and then the generated grid is transformed back to the physical space. Anisotropic stretching results in substantial reduction in the grid size. For example, stretching a grid in the wing spanwise direction would result in at least a factor-of-three reduction in the total number of grid points for a transport-type configuration. Grid stretching has been applied in the present problem extensively. Figure 7 shows part of the surface mesh on the wing where the triangles are highly stretched at the leading edges of the wing elements in the spanwise direction.

\section{GRID POST-PROCESSING}

Once the process of volume grid generation with VGRIDns is complete, some voids may remain in the inviscid portion of the grid which cannot be closed with the grid generator itself. Furthermore, the generated grid usually contains isolated pockets of distorted cells, which may be unacceptable to some solvers. These anomalies are created in the grid when the normal process of the advancing front is hampered due to, for example, distortion of the surface mesh and/or congestion of triangular faces on the front, especially when two fronts converge from opposite directions.

The inherent irregularity of unstructured grids not only provides ease of generation, but also offers flexibility for the alteration and post-processing of the generated grids. The lack of a regular structure in tetrahedral grids results in arbitrary cell groupings which, in effect, makes every portion of a grid independent of the rest. Consequently, any segment of a tetrahedral grid can be removed and locally re-meshed without disturbing the rest of the grid.

A computer program, referred to as POSTGRID, is used for post-processing of the generated grids for mesh quality improvement and/or completing an unfinished grid. The procedure is based on a local remeshing scheme that deletes distorted cells and creates empty pockets in the grid. In the case of an incomplete grid, one or more layers of cells are removed from the entangled fronts left by VGRIDns. After the anomalies are removed, the new fronts usually become smoother, making it easier to re-mesh the voids. The AFM is then utilized to fill the holes with a new set of tetrahedrons. All the post-processing operations are performed by the code automatically. Two criteria are employed to detect and remove distorted cells: tetrahedral cell volumes and included angles. The actual cell volumes are compared with the corresponding "ideal" volumes based on the local background grid characteristics, and the included angles of the tetrahedrons are compared against some preset values.

The local re-meshing technique with POSTGRID is very effective for the successful completion of grids as well as grid quality improvement and is routinely used in the VGRIDns system. The process is fast, automatic, and does not require user intervention. However, in rare cases where the surface mesh is severely distorted, some voids (usually those adjacent to the surface) 
persist and fail to close after repeated re-meshing attempts. Improving the quality of the surface mesh (by re-patching and/or modifying the local point distribution) often corrects the problem. Other useful features in POSTGRID include global smoothing of the volume grid by a Laplacian operation, moving grid capability, display

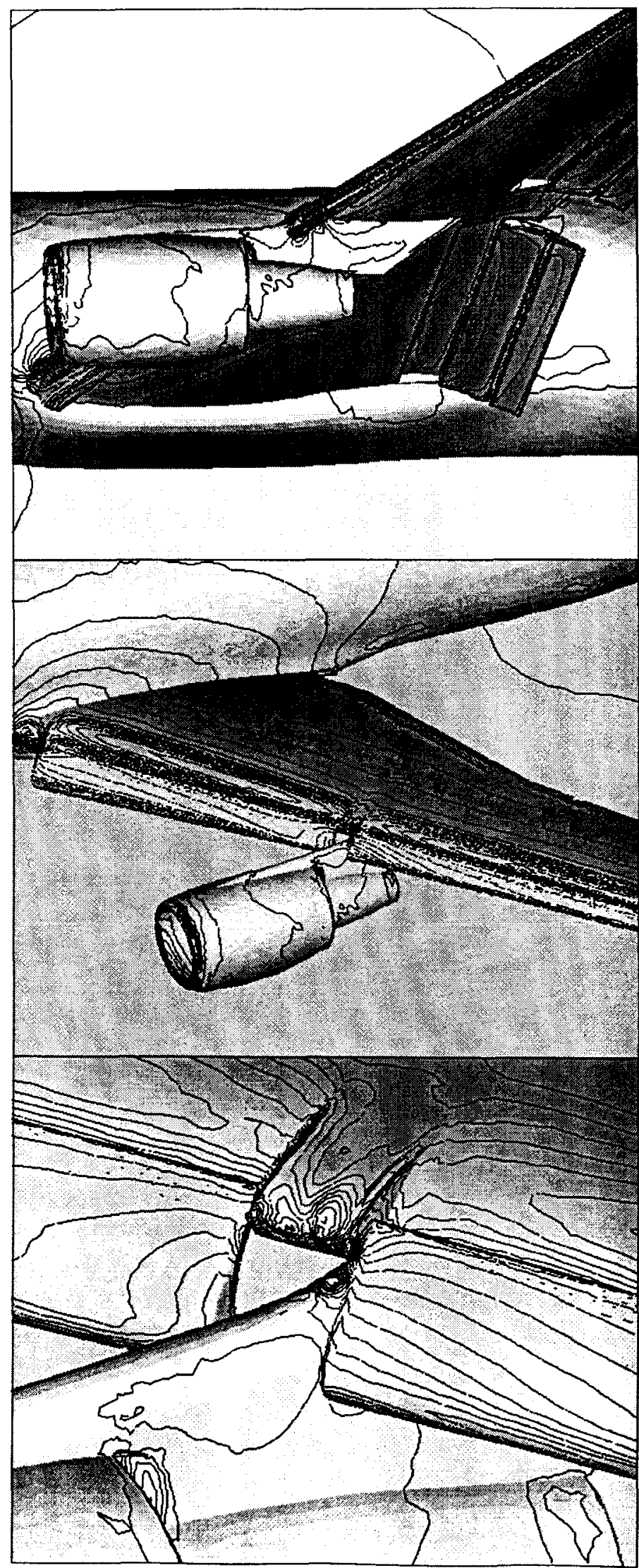

Figure 8. Computed surface pressure contours on EET. of volume grid cross-sections, and other useful graphics options.

\section{RESULT}

Extensive computational results are reported in Ref. [12] on a similar EET configuration (no nacelle and pylon) using grids generated with the present technique. The viscous flow computations, in that report, show excellent agreement with the experimental data. Navier-Stokes solutions have also been obtained on the present grid by Mavriplis and presented in a companion SAE paper ${ }^{13}$. The flow condition for the case studied is at a freestream Mach number of 0.2, a Reynolds number of 1.6 million based on the wing reference chord, and a flow incidence of 10 degrees.

Based on the above Reynolds number, the first layer spacing $\left(\delta_{1}\right)$ was prescribed as $1.35 \times 10^{-6}$ wing chord at the root, and the parameters $a$ and $b$ in Eq (1) were computed with a utility program with values 0.4 and 0.01 , respectively. The prescribed values have resulted in approximately 28 layers of tetrahedral cells in the boundary layer with a non-dimensional wall distance $\left(y^{+}\right)$ of about one off the surface. Anisotropic grid stretching was mainly applied at the leading and trailing edges of the main wing and flaps in the spanwise direction. The aspect ratios of the generated grid elements vary from a maximum value of about 100:1 (span:chord) at the midspan leading edges to $1: 1$ at the surface corners, fuselage, and in the field away from the geometry. These grid parameters resulted in a tetrahedral mesh containing 111,515 boundary nodes, 223,010 boundary triangles, $2,880,906$ total grid nodes, and $16,888,213$ tetrahedral cells.

The EET grid was entirely generated using a Silicon Graphics Octane workstation with a $195 \mathrm{MHZ}$ (R10000) processor. The entire process, from CAD definition to the final post-processed volume grid, was completed in about 68 cumulative labor hours by an expert. A large percentage (in this case, more than $90 \%$ ) of the total grid generation time was spent on the geometry preparation and the grid parameter setup. The surface and volume grids were automatically generated on the workstation in about 3.5 CPU hours.

To demonstrate the viability of the generated grid, and for completeness, three sample images depicting the computed surface pressure contours are illustrated in Fig. 8. Further details of the flow computation on the present grid can be found in Ref. [13].

\section{CONCLUDING REMARKS}

Based on the experiences gained during the course of this study and those of the previous investigators, the following conclusions are drawn in relation to the CFD analysis of high-lift problems.

1. While 2D flow analyses provide useful information for preliminary design, a realistic high-lift problem should be tackled in $3 D$ in order to obtain a true picture of the complex flowfield. 
2. Due to viscous domination of the flowfields involved, the N-S equations should be solved to better understand the flow characteristics.

3. Unstructured-grid CFD technology has reached the required maturity for analyzing realistic, $3 \mathrm{D}$ flows such as those encountered in the high-lift problems. Navier-Stokes solutions of these problems can now be generated in matter of days with the accuracy levels comparable to those of the structured grid methods.

4. Because of the large size of the computational grids involved (in the order of several million grid nodes) and the large computer memory required by the unstructured grid solvers, the parallel computation of high-lift problems is inevitable.

5. Multi-directional anisotropic grid stretching is a powerful technique for reducing the number of unstructured grid elements with no loss of the effective mesh resolution. The technique, employed in the present study, has confirmed that substantial computational economy is achievable with grid stretching without compromising the solution accuracy.

6. Another crucial technology for solving complex problems is the geometry/solution adaptive grid refinement. Automatic grid point insertion, based on surface curvature and flow solution characteristics, substantially enhances three fundamental elements in CFD: accuracy, automation, and efficiency. Without an automated grid adaptation algorithm, it becomes difficult to fulfill these critical requirements, especially for a complex problem such as high-lift.

7. For CFD to evolve into an essential component of the design process for high-lift configurations, a better link between the $C A D$ geometry definitions and the CFD grid generators is required. A large percentage of the grid generation time (days or even weeks) is often spent on the model repair and manipulation in order to redefine the geometry for grid generation. Such a time frame is simply unacceptable in a design environment.

Due to their importance, items 6 and 7 are among the priorities for further advancement of the technology. Work is currently in progress in these two areas at the NASA Langley Research Center. An ongoing study on the inviscid solution adaptive grids ${ }^{16}$ has demonstrated excellent potential for solving complex 3D problems. Extension of the method for adaptive viscous flow solutions and "geometry" adaptive grids (planned for future work) would substantially enhance the capability of the current technology for solving high-lift problems.

\section{ACKNOWLEDGMENTS}

The author would like to thank Dimitri Mavriplis, Institute for Computer Applications in Science and Engineering, NASA Langley Research Center, for providing the flow solutions presented in this paper.

\section{REFERENCES}

1. Anderson, K.W., "Navier-Stokes Computations and Experimental Comparisons for multi-element Airfoil Configurations," AIAA paper 93-0645, Jan. 1993.
2. Rogers, S.E., "Progress in High-Lift Aerodynamic Calculations," Journal of Aircraft, Vol. 31, No. 6, P12441251, Nov.-Dec. 1994.

3. Dominik, C., "Application of the Incompressible NavierStokes Equations to High-Lift Flows," AIAA Paper 941872, June 1994.

4. Mavriplis, D., "Turbulent Flow Calculations Using Unstructured and Adaptive Meshes," NASA CR-182102, 1990.

5. Dodbele, S.S., Hobbs, C.R., Kern, S.B., Ghee, T.A., Hall, D.R., and Ely, W.L., "Wind Tunnel Experiments and Navier-Stokes Computations of a High-Lift Military Airfoil," AIAA Paper 99-0540, January 1999.

6. Jones, K.M., Biedron, R.T., and Whitlock, M., "Application of a Navier-Stokes Solver to the Analysis of Multi-element Airfoils and Wings Using Multi-zonal Grid Techniques," AlAA Paper 95-1855, June 1995.

7. Takallu, M.A., and Laflin, K.R., "Reynolds-Average NavierStokes Simulations of Two Partial-Span Flap Wing Experiments," AIAA Paper 89-0701, January 1998.

8. Khorrami, M.R., Singer, B.A., Radeztsky, R.H., "Reynolds-Average Navier-Stokes Computations of a Flap Side-Edge Flow Field," AIAA Paper 89-0768, January 1998.

9. Dodbele, S.S., "Three Dimensional Aerodynamic Analysis of a High-Lift Transport Configuration," AIAA Paper 933536, August 1993.

10. Rogers, S.E., Cao, H.V., and Su, T.Y., "Grid Generation for Complex High-Lift Configurations," AIAA Paper 983011, June 1998.

11. Cao, H.V., Su, T.Y., and Rogers, S.E., "Navier-Stokes Analyses of a 747 High-Lift Configuration," AIAA Paper 982623, June 1998.

12. Mavriplis, D.J. and Pirzadeh S.Z., "Large-scale Parallel Unstructured Mesh Computations for 3D High-Lift Analysis," AIAA paper 99-0537, 1999.

13. Mavriplis, D.J., "Three-dimensional Viscous Flow Analysis for High-Lift Configurations Using a Parallel Unstructured Multigrid Solver," SAE 1999 World Aviation Congress and Exposition, paper 1999-01-5558, San Francisco, CA, October 19-21, 1999.

14. Pirzadeh, S., "Three Dimensional Viscous Grids by the Advancing-Layers Methods," AIAA Joumal, Vol. 34, No. 1, 1996, pp. 43-49.

15. Samareh, J., "GridTool: A Surface Modeling and Grid Generation Tool," Proceedings of the Workshop on Surface Modeling, Grid Generation, and Related Issues in CFD Solutions, NASA CP-3291, May 9-11, 1995.

16. Pirzadeh, S.Z., "An Adaptive Unstructured Grid Method by Grid Subdivision, Local Remeshing, and Grid Movement," AIAA Paper 99-3255, 1999.

17. Gatlin, G.M. and McGhee, R.J., "Experimental Investigation of Semi-span Model Testing Techniques", AIAA Joumal of Aircraft, Vol. 34, No. 4, 1997, pp. 500505.

18. Samareh, J.A., "Status and Future of Geometry Modeling and Grid Generation for Design and Optimization," Journal of Aircraft, Vol. 36, No. 1, 1999, pp. 97-104.

19. Pirzadeh, S., "Structured Background Grids for Generation of Unstructured Grids by Advancing Front Method," AIAA Joumal, Vol. 31, No. 2, 1993, pp. 257-265.

20. Löhner, R. and Parikh, P., "Generation of Three Dimensional Unstructured Grids by the Advancing Front Method," International Journal of Numerical Methods in Fluids, Vol. 8, 1988, pp. 1135-1149.

21. Pirzadeh, S., "Recent Progress in Unstructured Grid Generation," AIAA Paper 92-0445, January 1992.

22. Pirzadeh, S., "Progress Toward a User-Oriented Unstructured Viscous Grid Generator," AIAA Paper 960031, 1996. 